\title{
The birth of coronal holes
}

\author{
Fainshtein V.G. and G.V. Rudenko
}

Institute of solar-terrestrial physics SD RAS, 664033, Irkutsk-33, P.o.Box 4026, Russia email: vfain@iszf.irk.ru

\section{Introduction}

Coronal holes $(\mathrm{CHs})$ are large-scale regions of the corona with decreased density of material and temperatures. CHs are characterized by predominantly an open configuration of magnetic field lines. CHs are observed as regions of corona with decreased emission in soft X-rays, and appear simultaneously in different emission lines in distinct regions of solar atmosphere, Zirin (1988). CHs are known to be the source of the fast quasistationary solar wind (Nolte et al. 1976). At the same time answers to key questions which are related to the originating (the birth) of coronal holes till now are not obtained. It is known a little works in which the birth of CHs were investigated (Solodina et al. 1977; Bohlin \& Sheeley 1978; Watari et al. 1995). The objective of this paper is to study the birth of non-polar CHs. This paper continues our paper Fainshtein et al. (2002), in which one the birth of coronal holes was studied.

\section{Data and research methods}

We have studied the birth of CHs during 1999 - 2002 using daily NSO/KP coronal hole maps recorded in the line of HeI $\lambda 10830 \mathrm{~A}$ and FeXII $\lambda 195 \mathrm{~A}$ solar images (SOHO/EIT instrument). At that the dependencies of change areas and minimum brightness ( $\lambda 195$ A) of arising holes on the time were obtained. The configurations of the open magnetic tubes, associated with studied CHs, were obtained on the basis of calculations of an "instantaneous" distributions magnetic field in a corona within the framework of "potential field - source surface" model with using new Bd - technology (Rudenko 2001). The configurations of CHs in a line FeXII $\lambda 195$ A were determined with the help of the method similar to the method from the paper Stepanian and Malaschuk (2000).

\section{Results}

In items (a-f) the main results of analysis of $\mathrm{CH}$ birth are similar results of our paper Fainshtein et al. (2002) for period 1996 - 1999, and in items (g-k) the new results are presented.

(a) We have identified two classes of arising $\mathrm{CH}$ that were recorded in the line of $\mathrm{HeI}$ $\lambda 10830$ A: long-lived (lifetime 3 and more than day), and short-lived (lifetime 2 and less than day).

(b) An east-west and a north-south asymmetry was detected in the distribution of places of first recording of $\mathrm{CH}$ on the visible solar disk in the line of HeI $\lambda 10830 \mathrm{~A}$. The interpretation to these phenomena is adduced.

(c) It has been shown that, on average, the area of short-lived $\mathrm{CH}$ is smaller than that of long-lived holes at the time of their first recording.

(d) It has been found that birthplaces of short-lived $\mathrm{CH}$ are concentrated near to 
neutral lines of the large-scale photospheric magnetic field, while birthplaces of longlived holes tend to move away from these lines.

(e) It has been shown that most of new CHs produced near sunspot groups (active regions), whereas about $20 \%$ of holes are born at a relatively large distance from sunspot groups.

(f) It has been shown that the birth of long-lived CHs is almost not accompanied: (a) by solar flares in active regions adjacent the holes within ( -48 hours; +24 hours) from the time of first recording of $\mathrm{CH}$; and (b) by the disappearance of filaments in the immediate vicinity of the hole.

(g) During the period under review on observations of the Sun in the infrared line $\lambda 10830$ A the birth of any "major" CHs on the visual disk of the Sun was not revealed. Such CHs arose on a visible surface of the Sun, "coming up" behind of eastern limb owing to rotation of the Sun. Conditionally we have referred to major coronal holes of a hole, the area exceeded which one 250 square degrees.

(h) $90 \%$ of $\mathrm{CHs}$ which are born on observations of the Sun in an infrared line corresponded open magnetic tubes (OMTs). Was considered, that calculated OMT corresponds to $\mathrm{CH}$ if one of structures (CH or OMT) is completely inserted in other, or these structures are intercrossed or are contiguous to one another.

(i) Most of OMTs, associated with CHs, went out on a visual surface from behind of eastern limb. In remaining cases OMTs there were on the disk of the Sun for $1-5$ days before birth of $\mathrm{CHs}$ observed in a line of solar radiation $\lambda 10830 \mathrm{~A}$.

(j) Some cases of birth of $\mathrm{CHs}$ simultaneously in two lines of solar radiation $(\lambda 10830$ $\mathrm{A}$ and $\lambda 195 \mathrm{~A})$ are investigated. It is found that $\mathrm{CH}$ s registered in the line $\lambda 195 \mathrm{~A}$ there are on 1-3 days before the time of the first recording of $\mathrm{CHs}$ in a line $\lambda 10830 \mathrm{~A}$. It is revealed, that the process of birth a coronal hole in the line $\lambda 195$ A can wear "pulsatory" character: inside the arisen $\mathrm{CH}$ brighter structure filling the most part of $\mathrm{CH}$ can be formed, which one gradually (approximately during 1-1.5 day) darkens and disappeared, keeping in position new $\mathrm{CH}$.

(k) For several CHs (the line $\lambda 195 \mathrm{~A}$ the time dependencies of the area of a hole and minimum brightness of a radiation inside a hole are obtained. Is found, that the characteristic time of changes the indicated parameters up to its steady values comes to (10-25) hours (area of a hole) and (20-25) hours (minimum brightness) for studied events.

The work was done with support from the Federal Scientific and Technical Program "Astronomy" No 1105 and the government grant of financial support of RF leading scientific schools No 733.2003.2

\section{References}

Bohlin, J.D., \& Sheeley, N.R.Jr. 1978, Solar Physics, 56, 125-151.

Fainshtein, V.G., Rudenko, G.V., \& Grablevskii, S.V. 2002 In: The Solar-Terrestrial Physics,published by SB RAS, Issue 1 (114), 91-98.

Nolte J.T., Krieger, A.S., Timothy, A.F., Gold, R.E., Roelof, E.C., Vaiana, G., Lazarus, A.J., Sullivan, J.D., \& McIntosh, P.S. Solar Phys. 46, 303.

Rudenko, G.V. 2001 Solar Phys. 198, 5-30.

Solodina, G.V., Krieger, A.S. \& Nolte, J.T. 1977 Solar Phys. 54, 123-134.

Stepanian N.N. \& Malaschuk V.N. 2000 Izv. Krym. Astrofiz. Obs. 98, 8.

Watari, S., Kosuka, Y., Ohyama, M. \& Watanabe, T. 1995 J. Geomag. Geoelectr. 47 1063-1071.

Zirin, H. 1988 Cambridge University Press, New York, p. 259. 